\title{
A regularity criterion of the 3D MHD equations involving one velocity and one current density component in Lorentz space
}

\author{
Ravi P. Agarwal ${ }^{1}$, Sadek Gala ${ }^{2}$ and Maria Alessandra Ragusa ${ }^{2,3}$ \\ ${ }^{1}$ Department of Mathematics, Texas A\&M University-Kingsville, Kingsville, USA, \\ Ravi.Agarwal@tamuk.edu \\ ${ }^{2}$ Dipartimento di Matematica e Informatica, Università di Catania, \\ Viale Andrea Doria, 695125 Catania - Italy, \\ sgala793@gmail.com \\ ${ }^{3}$ RUDN University, 6 Miklukho - Maklay St, Moscow, 117198, Russia, \\ maragusa@dmi.unict.it
}

\begin{abstract} (3D) MHD equations. It is proved that the solution $(u, b)$ becomes regular provided that one velocity and one current density component of the solution satisfy$$
u_{3} \in L^{\frac{30 \alpha}{7 \alpha-45}}\left(0, T ; L^{\alpha, \infty}\left(\mathbb{R}^{3}\right)\right) \quad \text { with } \quad \frac{45}{7} \leq \alpha \leq \infty,
$$

and

$$
j_{3} \in L^{\frac{2 \beta}{2 \beta-3}}\left(0, T ; L^{\beta, \infty}\left(\mathbb{R}^{3}\right)\right) \quad \text { with } \quad \frac{3}{2} \leq \beta \leq \infty,
$$

which generalize some known results.
\end{abstract}

In this paper, we study the regularity criterion of weak solutions to the three-dimensional

Mathematics Subject Classification(2000): 35Q35, 35B65, 76D05.

Key words: MHD equations; regularity of weak solutions; Lorentz spaces. 


\section{Introduction}

This paper deals with the well-known problem of the regularity of the solutions for the 3D magneto-hydrodynamical (MHD) system

$$
\left\{\begin{array}{c}
\partial_{t} u+u \cdot \nabla u-(b \cdot \nabla) b-\Delta u+\nabla \pi=0, \\
\partial_{t} b+u \cdot \nabla b-b \cdot \nabla u-\Delta b=0, \\
\nabla \cdot u=\nabla \cdot b=0 \\
u(x, 0)=u_{0}(x), \quad b(x, 0)=b_{0}(x),
\end{array}\right.
$$

where $u=\left(u_{1}, u_{2}, u_{3}\right)$ is the velocity field, $b=\left(b_{1}, b_{2}, b_{3}\right)$ is the magnetic field, and $\pi$ is the scalar pressure, while $u_{0}$ and $b_{0}$ are the corresponding initial data satisfying $\nabla \cdot u_{0}=\nabla \cdot b_{0}=0$ in the sense of distribution.

Since Duvaut-Lions [9] and Sermange-Temam [25] constructed the so-called well-known weak solution $(u, b)(x, t)$ of the incompressible MHD equation for arbitrary $\left(u_{0}, b_{0}\right) \in L^{2}\left(\mathbb{R}^{3}\right)$ with $\nabla \cdot u_{0}(x)=\nabla \cdot b_{0}(x)=0$ in last century, the problem on the uniqueness and regularity of the weak solutions is one of the most challenging problem of the mathematical community. Hence, many researchers have developed different regularity criteria for the 3D MHD equations under assumption of certain growth conditions on the velocity or on the magnetic field (see, e.g., [7, 3, 4, 5, 8, 10, 14, 15, 16, 27, 28, 29, 30, 37, 38, and the references therein).

Recent years, the problem of so-called regularity criteria via one components was investigated for the MHD equations by some researchers (see 1, 11, 12, 13, 17, 18, 19, 20, 21, 23, 32, [33, 36] and the references therein). In particular, in 31, Yamazaki established the following regularity criterion by involving one velocity and one current density component, which shows that a weak solution $(u, b)$ is smooth on a time interval $(0, T]$ if

$$
u_{3} \in L^{p}\left(0, T ; L^{q}\left(\mathbb{R}^{3}\right)\right) \text { with } \frac{2}{p}+\frac{3}{q} \leq \frac{1}{3}+\frac{1}{2 q}, \quad \frac{15}{2}<q \leq \infty
$$

and

$$
j_{3} \in L^{p^{\prime}}\left(0, T ; L^{q^{\prime}}\left(\mathbb{R}^{3}\right)\right) \text { with } \frac{2}{p^{\prime}}+\frac{3}{q^{\prime}} \leq 2, \quad \frac{3}{2}<q^{\prime} \leq \infty,
$$

where $j_{3}$ is the third component of the current density $j=\nabla \times b=\left(j_{1}, j_{2}, j_{3}\right)$. Later, Zhang 34 improved the regularity criterion (1.2) to the following conditions

$$
u_{3} \in L^{p}\left(0, T ; L^{q}\left(\mathbb{R}^{3}\right)\right) \text { with } \frac{2}{p}+\frac{3}{q}=\frac{4}{9}-\frac{1}{3 q}, \frac{15}{2} \leq q \leq \infty,
$$

and

$$
j_{3} \in L^{p^{\prime}}\left(0, T ; L^{q^{\prime}}\left(\mathbb{R}^{3}\right)\right) \text { with } \frac{2}{p^{\prime}}+\frac{3}{q^{\prime}} \leq 2, \quad \frac{3}{2}<q^{\prime} \leq \infty .
$$

Very recently, this result (1.3) is further refined by Zhang [35] to prove the regularity criterion as long as the following conditions

$$
u_{3} \in L^{p}\left(0, T ; L^{q}\left(\mathbb{R}^{3}\right)\right) \text { with } \frac{2}{p}+\frac{3}{q}=\frac{4}{9}, \quad \frac{27}{4} \leq q \leq \infty,
$$


are satisfied.

Motivated by the papers [31, 34, 35, the purpose of the present paper is to refine (1.4) and to extend the above regularity criterion to the Lorentz space $L^{\alpha, \infty}$ which is larger than $L^{\alpha}$. More precisely, our main result now read as follows.

Theorem 1.1 Suppose $T>0,\left(u_{0}, b_{0}\right) \in L^{2}\left(\mathbb{R}^{3}\right)$ and $\nabla \cdot u_{0}=\nabla \cdot b_{0}=0$ in the sense of distributions. Assume that $(u, b)$ is a weak solution of the $3 D$ MHD equations (1.1) on $(0, T)$. If $u_{3}$ and $j_{3}$ satisfy the following growth conditions

$$
\int_{0}^{T}\left(\left\|u_{3}(\tau)\right\|_{L^{\alpha, \infty}}^{\frac{30 \alpha}{7 \alpha-45}}+\left\|j_{3}(\tau)\right\|_{L^{\beta, \infty}}^{\frac{2 \beta}{2 \beta-3}}\right) d \tau<\infty
$$

where $\frac{45}{7} \leq \alpha \leq \infty$ and $\frac{3}{2}<\beta \leq \infty$, then the weak solution $(u, b)$ is regular on $(0 ; T]$.

Remark 1.1 Theorem 1.1 extends the previous results on Navier-Stokes equations due to the fact that the MHD equations with $b(x, t)=0$ reduces the Navier-Stokes equations. According to the embedding relation $L^{\alpha} \subseteq L^{\alpha, \infty}$, it is easy to see that our result of Theorem 1.1 is an improvement of the recent works by Yamazaki [31] and Zhang [34, 35].

\section{Preliminaries}

Throughout this paper, we use the following usual notations. $L^{p}\left(\mathbb{R}^{3}\right)$ denotes the Lebegue space associated with norm

$$
\|f\|_{L^{p}}=\left\{\begin{aligned}
\left(\int_{\mathbb{R}^{3}}|f(x)|^{p} d x\right)^{\frac{1}{p}}, & \text { for } \quad 1 \leq p<\infty \\
\underset{x s \mathbb{R}^{3}}{e s \sup }|f(x)|, & \text { for } \quad p=\infty
\end{aligned}\right.
$$

$H^{k}\left(\mathbb{R}^{3}\right)$ denotes the Hilbert space $\left\{u \in L^{2}\left(\mathbb{R}^{3}\right):\left\|\nabla^{k} u\right\|_{L^{2}}<\infty\right\}$. Let $(X, \mathcal{M}, \mu)$ be a nonatomic measurable space. For a complex- or real-valued $\mu$-measurable function $f(x)$ defined on $X$, its distributional function is defined by

$$
f_{*}(\sigma)=\mu\{x \in X: f(x)>\sigma\}, \quad \text { for } \sigma>0,
$$

which is non-increasing and continuous from the right. Furthermore, its non-increasing rearrangement $f^{*}$ is defined by

$$
f^{*}(t)=\inf \left\{s>0: f_{*}(s) \leq t\right\}, \quad \text { for } t>0,
$$

which is also non-increasing and continuous from the right and has the same distributional function as $f(x)$.

The Lorentz space $L^{p, q}$ on $(X, \mathcal{M}, \mu)$ is the collection of all real- or complex-valued $\mu$-measurable functions $f(x)$ defined on $X$ such that $\|f\|_{L^{p, q}}<\infty$, where

$$
\|f\|_{L^{p, q}}=\left\{\begin{array}{c}
\left(\frac{q}{p} \int_{0}^{\infty}\left(t^{\frac{1}{p}} f^{*}(t)\right)^{q} \frac{d t}{t}\right)^{\frac{1}{q}}, \quad \text { if } 1 \leq p<\infty, \quad 1<q<\infty \\
\sup _{t>0}\left(t^{\frac{1}{p}} f^{*}(t)\right), \quad \text { if } 1 \leq p<\infty, \quad q=\infty .
\end{array}\right.
$$


Moreover,

$$
\|f\|_{L^{p, \infty}}=\sup _{t>0}\left(t^{\frac{1}{p}} f^{*}(t)\right)=\sup _{\sigma>0} \sigma\left(f_{*}(\sigma)\right)^{\frac{1}{p}}
$$

for any $f \in L^{p, \infty}$. For details, we refer to [2] and [26].

The space definition implies the following continuous embeddings:

$$
L^{p}\left(\mathbb{R}^{3}\right)=L^{p, p}\left(\mathbb{R}^{3}\right) \hookrightarrow L^{p, q}\left(\mathbb{R}^{3}\right) \hookrightarrow L^{p, \infty}\left(\mathbb{R}^{3}\right), \quad 1 \leq p \leq q<\infty .
$$

In order to prove Theorem 1.1, we need the following Hölder inequality in Lorentz spaces (see, e.g., O'Neil [24] and [22]).

Lemma 2.1 ([24], Theorems 3.4 and 3.5) Let $f \in L^{p_{2}, q_{2}}\left(\mathbb{R}^{3}\right)$ and $g \in L^{p_{3}, q_{3}}\left(\mathbb{R}^{3}\right)$ with $1 \leq$ $p_{2}, p_{3} \leq \infty, 1 \leq q_{2}, q_{3} \leq \infty$. Then $f g \in L^{p_{1}, q_{1}}\left(\mathbb{R}^{3}\right)$ with

$$
\frac{1}{p_{1}}=\frac{1}{p_{2}}+\frac{1}{p_{3}}, \frac{1}{q_{1}}=\frac{1}{q_{2}}+\frac{1}{q_{3}}
$$

and the Hölder inequality of Lorentz spaces

$$
\|f g\|_{L^{p_{1}, q_{1}}} \leq C\|f\|_{L^{p_{2}, q_{2}}}\|g\|_{L^{p_{3}, q_{3}}}
$$

holds true for a positive constant $C$.

We also recall Gagliardo-Nirenberg's inequality in Lorentz spaces which plays an important role in the proofs of Theorem 1.1

Lemma 2.2 Let $f \in L^{p, q}\left(\mathbb{R}^{3}\right)$ with $1 \leq p, q, p_{4}, q_{4}, p_{5}, q_{5} \leq \infty$. Then the Gagliardo-Nirenberg inequality of Lorentz spaces

$$
\|f\|_{L^{p, q}} \leq C\|f\|_{L^{p_{4}, q_{4}}}^{\theta}\|f\|_{L^{p_{5}, q_{5}}}^{1-\theta}
$$

holds for a positive constant $C$ and

$$
\frac{1}{p}=\frac{\theta}{p_{4}}+\frac{1-\theta}{p_{5}}, \quad \frac{1}{q}=\frac{\theta}{q_{4}}+\frac{1-\theta}{q_{5}}, \quad \theta \in(0,1) .
$$

\section{Proof of main result.}

In this section, under the assumptions of the Theorem 1.1, we prove our main result. Before proving our result, we recall the following muliplicative Sobolev imbedding inequality in the whole space $\mathbb{R}^{3}$ (see, for example [6]) :

$$
\|f\|_{L^{6}} \leq C\left\|\nabla_{h} f\right\|_{L^{2}}^{\frac{2}{3}}\left\|\partial_{3} f\right\|_{L^{2}}^{\frac{1}{3}}
$$

where $\nabla_{h}=\left(\partial_{x_{1}}, \partial_{x_{2}}\right)$ is the horizontal gradient operator. We are now give the proof of our main theorem.

Proof: To prove our result, it suffices to show that for any fixed $T>T^{*}$, there holds

$$
\sup _{0 \leq t \leq T^{*}}\|\nabla u(t)\|_{L^{2}}^{2}+\|\nabla b(t)\|_{L^{2}}^{2} \leq C_{T},
$$


where $T^{*}$, which denotes the maximal existence time of a strong solution and $C_{T}$ is an absolute constant which only depends on $T, u_{0}$ and $b_{0}$.

The method of our proof is the standard energy estimates as in 31. We will based on two major parts. The first one establishes the bounds of $\left(\left\|\nabla_{h} u\right\|_{L^{2}}^{2}+\left\|\nabla_{h} b\right\|_{L^{2}}^{2}\right)$, while the second gives the bounds of the $H^{1}$-norm of velocity $u$ and magnetic field $b$ in terms of the results of part one.

For this purpose, we multiply the first and second equations of (1.1) by $-\Delta_{h} u$ and $-\Delta_{h} b$, respectively, and integrate them over $\mathbb{R}^{3}$ with respect to the spatial variable. Then, integration by parts gives the following identity:

$$
\begin{aligned}
& \frac{1}{2} \frac{d}{d t}\left(\left\|\nabla_{h} u\right\|_{L^{2}}^{2}+\left\|\nabla_{h} b\right\|_{L^{2}}^{2}\right)+\left\|\nabla \nabla_{h} u\right\|_{L^{2}}^{2}+\left\|\nabla \nabla_{h} b\right\|_{L^{2}}^{2} \\
= & \int_{\mathbb{R}^{3}}(u \cdot \nabla) u \cdot \Delta_{h} u d x-\int_{\mathbb{R}^{3}}(b \cdot \nabla) b \cdot \Delta_{h} u d x \\
& +\int_{\mathbb{R}^{3}}(u \cdot \nabla) b \cdot \Delta_{h} b d x-\int_{\mathbb{R}^{3}}(b \cdot \nabla) u \cdot \Delta_{h} b d x \\
= & \text { RHS, }
\end{aligned}
$$

where $\Delta_{h}=\partial_{x_{1}}^{2}+\partial_{x_{2}}^{2}$ is the horizontal Laplacian. For simplicity of exposition, we denote

$$
\begin{aligned}
\mathcal{L}^{2}(t) & =\sup _{\tau \in[\Gamma, t]}\left(\left\|\nabla_{h} u(\tau)\right\|_{L^{2}}^{2}+\left\|\nabla_{h} b(\tau)\right\|_{L^{2}}^{2}\right)+\int_{\Gamma}^{t}\left(\left\|\nabla \nabla_{h} u(\tau)\right\|_{L^{2}}^{2}+\left\|\nabla \nabla_{h} b(\tau)\right\|_{L^{2}}^{2}\right) d \tau, \\
\mathcal{J}^{2}(t) & =\sup _{\tau \in[\Gamma, t]}\left(\|\nabla u(\tau)\|_{L^{2}}^{2}+\|\nabla b(\tau)\|_{L^{2}}^{2}\right)+\int_{\Gamma}^{t}\left(\|\Delta u(\tau)\|_{L^{2}}^{2}+\|\Delta b(\tau)\|_{L^{2}}^{2}\right) d \tau,
\end{aligned}
$$

for $t \in\left[\Gamma, T^{*}\right)$. We choose $\epsilon, \eta>0$ to be precisely determined subsequently and then select $\Gamma<T^{*}$ sufficiently close to $T^{*}$ such that for all $\Gamma \leq t<T^{*}$,

$$
\int_{\Gamma}^{t}\left(\|\nabla u(\tau)\|_{L^{2}}^{2}+\|\nabla b(\tau)\|_{L^{2}}^{2}\right) d \tau \leq \epsilon \ll 1 \quad \text { and } \quad \int_{\Gamma}^{t}\left\|j_{3}(\tau)\right\|_{L^{\beta}}^{\frac{2 \beta}{2 \beta-3}} d \tau \leq \eta \ll 1 .
$$

Applying the divergence-free condition, $\nabla \cdot u=\nabla \cdot b=0$, we find that RHS can be estimated as

$$
\begin{aligned}
\mathrm{RHS} \leq & \int_{\mathbb{R}^{3}}\left|u_{3}\right||\nabla u|\left|\nabla \nabla_{h} u\right| d x+\int_{\mathbb{R}^{3}}\left|u_{3}\right||\nabla b|\left|\nabla \nabla_{h} b\right| d x+\int_{\mathbb{R}^{3}}\left|b_{3}\right||\nabla u|\left|\nabla \nabla_{h} b\right| d x \\
& +\int_{\mathbb{R}^{3}}\left|b_{3}\right||\nabla b|\left|\nabla \nabla_{h} u\right| d x+\int_{\mathbb{R}^{3}}\left|\nabla_{h} u\right|\left|\nabla_{h} b\right|\left|j_{3}\right| d x \\
= & L_{1}+L_{2}+L_{3}+L_{4}+L_{5},
\end{aligned}
$$

where the last inequality was proved in [31. (see, Proposition 3.1 in 31] for details).

With the use of the Lemma 2.1] (3.1), and the Young inequality, we derive the estimate of the first term $L_{1}$ of (3.3) as follows :

$$
\begin{aligned}
L_{1} & \leq C\left\|u_{3}\right\|_{L^{\alpha, \infty}}\|\nabla u\|_{L^{\frac{2 \alpha}{\alpha-2}, 2}}\left\|\nabla \nabla_{h} u\right\|_{L^{2}} \\
& \leq C\left\|u_{3}\right\|_{L^{\alpha, \infty}}\|\nabla u\|_{L^{2}}^{1-\frac{3}{\alpha}}\|\nabla u\|_{L^{6}}^{\frac{3}{\alpha}}\left\|\nabla \nabla_{h} u\right\|_{L^{2}} \\
& \leq C\left\|u_{3}\right\|_{L^{\alpha, \infty}}\|\nabla u\|_{L^{2}}^{1-\frac{3}{\alpha}}\|\Delta u\|_{L^{2}}^{\frac{1}{\alpha}}\left\|\nabla \nabla_{h} u\right\|_{L^{2}}^{1+\frac{2}{\alpha}} \\
& \leq C\left\|u_{3}\right\|_{L^{\alpha, \infty}}^{\frac{2 \alpha}{\alpha-2}}\|\nabla u\|_{L^{2}}^{2-\frac{2}{\alpha-2}}\|\Delta u\|_{L^{2}}^{\frac{2}{\alpha-2}}+\frac{1}{8}\left\|\nabla \nabla_{h} u\right\|_{L^{2}}^{2},
\end{aligned}
$$


where we have used the following Gagliardo-Nirenberg inequality in Lorentz spaces :

$$
\|\nabla u\|_{L^{\frac{2 \alpha}{\alpha-2}, 2}} \leq C\|\nabla u\|_{L^{2}}^{1-\frac{3}{\alpha}}\|\nabla u\|_{L^{6}}^{\frac{3}{\alpha}} .
$$

Similarly, employing the Hölder inequality and the Gagliardo-Nirenberg inequality give that for $L_{1}$,

$$
L_{2} \leq C\left\|u_{3}\right\|_{L^{\alpha, \infty}}^{\frac{2 \alpha}{\alpha-2}}\|\nabla b\|_{L^{2}}^{2-\frac{2}{\alpha-2}}\|\Delta b\|_{L^{2}}^{\frac{2}{\alpha-2}}+\frac{1}{8}\left\|\nabla \nabla_{h} b\right\|_{L^{2}}^{2} .
$$

We now estimate $L_{3}$,

$$
\begin{aligned}
L_{3} & \leq\left\|b_{3}\right\|_{L^{10}}\|\nabla u\|_{L^{\frac{5}{2}}}\left\|\nabla \nabla_{h} b\right\|_{L^{2}} \leq C\left\|b_{3}\right\|_{L^{10}}\|\nabla u\|_{L^{2}}^{\frac{7}{10}}\|\nabla u\|_{L^{6}}^{\frac{3}{10}}\left\|\nabla \nabla_{h} b\right\|_{L^{2}} \\
& \leq C\left\|b_{3}\right\|_{L^{\frac{10}{3}}}\|\nabla u\|_{L^{2}}^{\frac{7}{10}}\left\|\nabla \nabla_{h} u\right\|_{L^{2}}^{\frac{1}{5}}\|\Delta u\|_{L^{2}}^{\frac{1}{10}}\left\|\nabla \nabla_{h} b\right\|_{L^{2}} \\
& \leq C\left\|b_{3}\right\|_{L^{10}}^{\frac{5}{2}}\|\nabla u\|_{L^{2}}^{\frac{7}{4}}\|\Delta u\|_{L^{2}}^{\frac{1}{4}}+\frac{1}{8}\left(\left\|\nabla \nabla_{h} b\right\|_{L^{2}}^{2}+\left\|\nabla \nabla_{h} u\right\|_{L^{2}}^{2}\right),
\end{aligned}
$$

where we have used the fact $\|\nabla u\|_{L^{\frac{5}{2}}} \leq C\|\nabla u\|_{L^{2}}^{\frac{7}{10}}\|\nabla u\|_{L^{6}}^{\frac{3}{10}}$.

Likewise,

$$
L_{4} \leq C\left\|b_{3}\right\|_{L^{10}}^{\frac{5}{2}}\|\nabla b\|_{L^{2}}^{\frac{7}{4}}\|\Delta b\|_{L^{2}}^{\frac{1}{4}}+\frac{1}{8}\left(\left\|\nabla \nabla_{h} b\right\|_{L^{2}}^{2}+\left\|\nabla \nabla_{h} u\right\|_{L^{2}}^{2}\right) .
$$

For $L_{5}$, by applying the Hölder inequality, the Gagliardo-Nirenberg inequality and the Young inequality, one shows that

$$
\begin{aligned}
L_{5} & =\int_{\mathbb{R}^{3}}\left|\nabla_{h} u\right|\left|\nabla_{h} b\right|\left|j_{3}\right| d x \leq \frac{1}{2} \int_{\mathbb{R}^{3}}\left(\left|\nabla_{h} u\right|^{2}+\left|\nabla_{h} b\right|^{2}\right)\left|j_{3}\right| d x \\
& \leq C\left\|j_{3}\right\|_{L^{\beta, \infty}}\left(\left\|\nabla_{h} u\right\|_{L^{\frac{2 \beta}{\beta-2}, 2}}\left\|\nabla_{h} u\right\|_{L^{2}}+\left\|\nabla_{h} u\right\|_{L^{\frac{2 \beta}{\beta-2}, 2}}\left\|\nabla_{h} b\right\|_{L^{2}}\right) \\
& \leq C\left\|j_{3}\right\|_{L^{\beta, \infty}}\left(\left\|\nabla_{h} u\right\|_{L^{2}}^{2-\frac{3}{\beta}}\left\|\nabla \nabla_{h} u\right\|_{L^{2}}^{\frac{3}{s}}+\left\|\nabla_{h} b\right\|_{L^{2}}^{2-\frac{3}{\beta}}\left\|\nabla \nabla_{h} b\right\|_{L^{2}}^{\frac{3}{s}}\right) \\
& \leq C\left\|j_{3}\right\|_{L^{\beta, \infty}}^{\frac{2 \beta}{2 \beta-3}}\left(\left\|\nabla_{h} u\right\|_{L^{2}}^{2}+\left\|\nabla_{h} b\right\|_{L^{2}}^{2}\right)+\frac{1}{8}\left(\left\|\nabla \nabla_{h} b\right\|_{L^{2}}^{2}+\left\|\nabla \nabla_{h} u\right\|_{L^{2}}^{2}\right) .
\end{aligned}
$$

Inserting all the estimates into (3.3), Gronwall's type argument using

$$
1 \leq \sup _{\lambda \in[\Gamma, \tau]} \exp \left(c \int_{\lambda}^{\tau}\left\|j_{3}(\varphi)\right\|_{L^{\beta, \infty}}^{\frac{2 \beta}{2 \beta-3}} d \varphi\right) \lesssim \exp \left(c \int_{0}^{T^{*}}\left\|j_{3}(\varphi)\right\|_{L^{\beta, \infty}}^{\frac{2 \beta}{2 \beta-3}} d \varphi\right) \lesssim 1
$$

due to (1.5) leads to, for every $\tau \in[\Gamma, t]$

$$
\begin{aligned}
\mathcal{L}^{2}(t) \leq & C+C \int_{\Gamma}^{t}\left\|u_{3}\right\|_{L^{\alpha, \infty}}^{\frac{2 \alpha}{\alpha-2}}\left(\|\nabla u\|_{L^{2}}^{2-\frac{2}{\alpha-2}}\|\Delta u\|_{L^{2}}^{\frac{2}{\alpha-2}}+\|\nabla b\|_{L^{2}}^{2-\frac{2}{\alpha-2}}\|\Delta b\|_{L^{2}}^{\frac{2}{\alpha-2}}\right) d \tau \\
& +C \int_{\Gamma}^{t}\left\|b_{3}\right\|_{L^{10}}^{\frac{5}{2}}\left(\|\nabla u\|_{L^{2}}^{\frac{7}{4}}\|\Delta u\|_{L^{2}}^{\frac{1}{4}}+\|\nabla b\|_{L^{2}}^{\frac{7}{4}}\|\Delta b\|_{L^{2}}^{\frac{1}{4}}\right) d \tau \\
& +C \int_{\Gamma}^{t}\left\|j_{3}\right\|_{L^{\beta, \infty}}^{\frac{2 \beta}{2 \beta-3}}\left(\left\|\nabla_{h} u\right\|_{L^{2}}^{2}+\left\|\nabla_{h} b\right\|_{L^{2}}^{2}\right) d \tau \\
= & C+\mathcal{I}_{1}(t)+\mathcal{I}_{2}(t)+\mathcal{I}_{3}(t) .
\end{aligned}
$$

Next, we analyze the right-hand side of (3.4) one by one. First, due to (3.2) and the definition 
of $\mathcal{J}^{2}$, we have

$$
\begin{aligned}
& \mathcal{I}_{1}(t) \leq C\left(\sup _{\tau \in[\Gamma, t]}\|\nabla u(\tau)\|_{L^{2}}^{\frac{3}{2}-\frac{2}{\alpha-2}}\right) \int_{\Gamma}^{t}\left\|u_{3}(\tau)\right\|_{L^{\alpha, \infty}}^{\frac{2 \alpha}{\alpha-2}}\|\nabla u(\tau)\|_{L^{2}}^{\frac{1}{2}}\|\Delta u(\tau)\|_{L^{2}}^{\frac{2}{\alpha-2}} d \tau \\
& +C\left(\sup _{\tau \in[\Gamma, t]}\|\nabla b(\tau)\|_{L^{2}}^{\frac{3}{2}-\frac{2}{\alpha-2}}\right) \int_{\Gamma}^{t}\left\|u_{3}(\tau)\right\|_{L^{\alpha, \infty}}^{\frac{2 \alpha}{\alpha-2}}\|\nabla b(\tau)\|_{L^{2}}^{\frac{1}{2}}\|\Delta b(\tau)\|_{L^{2}}^{\frac{2}{\alpha-2}} d \tau \\
& \leq C \mathcal{J}^{\frac{3}{2}-\frac{2}{\alpha-2}}(t)\left(\int_{\Gamma}^{t}\left\|u_{3}(\tau)\right\|_{L^{\alpha, \infty}}^{\frac{8 \alpha}{3 \alpha-10}} d \tau\right)^{\frac{3}{4}-\frac{1}{\alpha-2}}\left(\int_{\Gamma}^{t}\|\nabla u(\tau)\|_{L^{2}}^{2} d \tau\right)^{\frac{1}{4}}\left(\int_{\Gamma}^{t}\|\Delta u(\tau)\|_{L^{2}}^{2} d \tau\right)^{\frac{1}{\alpha-2}} \\
& +C \mathcal{J}^{\frac{3}{2}-\frac{2}{\alpha-2}}(t)\left(\int_{\Gamma}^{t}\left\|u_{3}(\tau)\right\|_{L^{\alpha, \infty}}^{\frac{8 \alpha}{3 \alpha-10}} d \tau\right)^{\frac{3}{4}-\frac{1}{\alpha-2}}\left(\int_{\Gamma}^{t}\|\nabla b(\tau)\|_{L^{2}}^{2} d \tau\right)^{\frac{1}{4}}\left(\int_{\Gamma}^{t}\|\Delta b(\tau)\|_{L^{2}}^{2} d \tau\right)^{\frac{1}{\alpha-2}} \\
& \leq C \mathcal{J}^{\frac{3}{2}-\frac{2}{\alpha-2}}(t)\left(\int_{\Gamma}^{t}\left\|u_{3}(\tau)\right\|_{L^{\alpha, \infty}}^{\frac{8 \alpha}{3 \alpha-10}} d \tau\right)^{\frac{3}{4}-\frac{1}{\alpha-2}} \epsilon^{\frac{1}{4}} \mathcal{J}^{\frac{2}{\alpha-2}}(t) \\
& =C \epsilon^{\frac{1}{4}} \mathcal{J}^{\frac{3}{2}}(t)\left(\int_{\Gamma}^{t}\left\|u_{3}(\tau)\right\|_{L^{\alpha, \infty}}^{\frac{8 \alpha}{3 \alpha-10}} d \tau\right)^{\frac{3}{4}-\frac{1}{\alpha-2}} .
\end{aligned}
$$

Now, we estimate the term $\mathcal{I}_{2}(t)$ as

$$
\begin{aligned}
\mathcal{I}_{2}(t) \leq & C\left(\sup _{\tau \in[\Gamma, t]}\left\|b_{3}(\tau)\right\|_{L^{10}}^{\frac{5}{2}}\right) \int_{\Gamma}^{t}\|\nabla u(\tau)\|_{L^{2}}^{\frac{7}{4}}\|\Delta u(\tau)\|_{L^{2}}^{\frac{1}{4}} d \tau \\
& +\left(\sup _{\tau \in[\Gamma, t]}\left\|b_{3}(\tau)\right\|_{L^{10}}^{\frac{5}{2}}\right) \int_{\Gamma}^{t}\|\nabla b(\tau)\|_{L^{2}}^{\frac{7}{4}}\|\Delta b(\tau)\|_{L^{2}}^{\frac{1}{4}} d \tau \\
\leq & \left(\sup _{\tau \in[\Gamma, t]}\left\|b_{3}(\tau)\right\|_{L^{10}}^{\frac{5}{2}}\right)\left(\int_{\Gamma}^{t}\|\nabla u(\tau)\|_{L^{2}}^{2} d \tau\right)^{\frac{7}{8}}\left(\int_{\Gamma}^{t}\|\Delta u(\tau)\|_{L^{2}}^{2} d \tau\right)^{\frac{1}{8}} \\
& +\left(\sup _{\tau \in[\Gamma, t]}\left\|b_{3}(\tau)\right\|_{L^{10}}^{\frac{5}{2}}\right)\left(\int_{\Gamma}^{t}\|\nabla b(\tau)\|_{L^{2}}^{2} d \tau\right)^{\frac{7}{8}}\left(\int_{\Gamma}^{t}\|\Delta b(\tau)\|_{L^{2}}^{2} d \tau\right)^{\frac{1}{8}} \\
\leq & C\left(\sup _{\tau \in[\Gamma, t]}\left\|b_{3}(\tau)\right\|_{L^{10}}^{\frac{5}{2}}\right) \epsilon^{\frac{7}{8}} \mathcal{J}^{\frac{1}{4}}(t) .
\end{aligned}
$$

For $\mathcal{I}_{3}(t)$, applying Hölder's and Young's inequalities, we get

$$
\begin{aligned}
\mathcal{I}_{3}(t) & \leq C \sup _{\tau \in[\Gamma, t]}\left(\left\|\nabla_{h} u(\tau)\right\|_{L^{2}}^{2}+\left\|\nabla_{h} b(\tau)\right\|_{L^{2}}^{2}\right) \int_{\Gamma}^{t}\left\|j_{3}(\tau)\right\|_{L^{\beta, \infty}}^{\frac{2 \beta}{2 \beta-3}} d \tau \\
& \leq C \eta \mathcal{L}^{2}(t) .
\end{aligned}
$$

Therefore, combining the estimates of $\mathcal{I}_{1}(t), \mathcal{I}_{2}(t)$ and $\mathcal{I}_{3}(t)$ together with (3.4) and taking $\eta$ small enough, it is easy to see that for all $\Gamma \leq t<T^{*}$ :

$$
\mathcal{L}^{2}(t) \leq C+C \epsilon^{\frac{1}{4}} \mathcal{J}^{\frac{3}{2}}(t)\left(\int_{\Gamma}^{t}\left\|u_{3}(\tau)\right\|_{L^{\alpha, \infty}}^{\frac{8 \alpha}{3 \alpha-10}} d \tau\right)^{\frac{3 \alpha-10}{4(\alpha-2)}}+C\left(\sup _{\tau \in[\Gamma, t]}\left\|b_{3}(\tau)\right\|_{L^{10}}^{\frac{5}{2}}\right) \epsilon^{\frac{7}{8}} \mathcal{J}^{\frac{1}{4}}(t)
$$

Now, we will establish the bounds of $L^{10}$-norm of the magnetic field $b_{3}$. In order to do it, we recall the third equation of the magnetic field:

$$
\partial_{t} b_{3}-\Delta b_{3}+(u \cdot \nabla) b_{3}=(b \cdot \nabla) u_{3}
$$


and multiply this equation by $\left|b_{3}\right|^{8} b_{3}$, integrating by parts, using incompressibility conditions to obtain

$$
\begin{aligned}
\frac{1}{10} \frac{d}{d t} \int_{\mathbb{R}^{3}}\left|b_{3}\right|^{10} d x+\frac{9}{25} \int_{\mathbb{R}^{3}}\left|\nabla\left(b_{3}^{5}\right)\right|^{2} d x & =\int_{\mathbb{R}^{3}}\left(b \cdot \nabla u_{3}\right)\left(\left|b_{3}\right|^{8} b_{3}\right) d x \\
& =-9 \int_{\mathbb{R}^{3}} b \cdot\left|b_{3}\right|^{4}\left(\left|b_{3}\right|^{4} \nabla b_{3}\right) u_{3} d x \\
& \leq \frac{9}{5} \int_{\mathbb{R}^{3}}|b|\left(\left|b_{3}\right|^{5}\right)^{\frac{4}{5}}\left|u_{3}\right|\left|\nabla\left(b_{3}^{5}\right)\right| d x=I .
\end{aligned}
$$

Using the Hölder, Young inequalities and interpolation, the estimates of $I$ is given by

$$
\begin{aligned}
& I \leq \frac{9}{5}\|b\|_{L^{6}}\left\|u_{3}\left(\left|b_{3}\right|^{5}\right)^{\frac{4}{5}}\right\|_{L^{3}}\left\|\nabla\left(b_{3}^{5}\right)\right\|_{L^{2}} \\
& \leq C\left\|\nabla_{h} b\right\|_{L^{2}}^{\frac{2}{3}}\|\nabla b\|_{L^{2}}^{\frac{1}{3}}\left\|u_{3}\right\|_{L^{\alpha, \infty}}\left\|\left(\left|b_{3}\right|^{5}\right)^{\frac{4}{5}}\right\|_{L^{\frac{3 \alpha}{\alpha-3}, 3}}\left\|\nabla\left(b_{3}^{5}\right)\right\|_{L^{2}} \\
& \leq C\left\|\nabla_{h} b\right\|_{L^{2}}^{\frac{2}{3}}\|\nabla b\|_{L^{2}}^{\frac{1}{3}}\left\|u_{3}\right\|_{L^{\alpha, \infty}}\left\|\left(\left|b_{3}\right|^{5}\right)^{\frac{4}{5}}\right\|_{L^{\frac{3 \alpha}{\alpha-3}, 2}}\left\|\nabla\left(b_{3}^{5}\right)\right\|_{L^{2}} \\
& \leq C\left\|\nabla_{h} b\right\|_{L^{2}}^{\frac{2}{3}}\|\nabla b\|_{L^{2}}^{\frac{1}{3}}\left\|u_{3}\right\|_{L^{\alpha, \infty}}\left(\left\|b_{3}^{5}\right\|_{L^{2}}^{\frac{3(\alpha-5)}{4 \alpha}}\left\|\nabla\left(b_{3}^{5}\right)\right\|_{L^{2}}^{\frac{15+\alpha}{4 \alpha}}\right)^{\frac{4}{5}}\left\|\nabla\left(b_{3}^{5}\right)\right\|_{L^{2}} \\
& \leq C\left\|\nabla_{h} b\right\|_{L^{2}}^{\frac{20 \alpha}{3(4 \alpha-15)}}\|\nabla b\|_{L^{2}}^{\frac{10 \alpha}{3(\alpha-15)}}\left\|u_{3}\right\|_{L^{\alpha}}^{\frac{10 \alpha}{4 \alpha-15}}\left\|b_{3}^{5}\right\|_{L^{2}}^{\frac{6(\alpha-5)}{4 \alpha-15}}+\frac{9}{5}\left\|\nabla\left(b_{3}^{5}\right)\right\|_{L^{2}}^{2}
\end{aligned}
$$

Putting $I$ in (3.6), we get

$$
\frac{d}{d t} \int_{\mathbb{R}^{3}}\left|b_{3}\right|^{10} d x \leq C\left\|\nabla_{h} b\right\|_{L^{2}}^{\frac{20 \alpha}{3(4-15)}}\|\nabla b\|_{L^{2}}^{\frac{10 \alpha}{3(\alpha-15)}}\left\|u_{3}\right\|_{L^{\alpha}}^{\frac{10 \alpha}{4 \alpha-15}}\left\|b_{3}\right\|_{L^{10}}^{\frac{30(\alpha-5)}{4 \alpha-15}} .
$$

Dividing by $\left\|b_{3}\right\|_{L^{10}}^{\frac{30(\alpha-5)}{4 \alpha-15}}$, we arrive at

$$
\frac{d}{d t}\left\|b_{3}\right\|_{L^{10}}^{\frac{10 \alpha}{4 \alpha-15}} \leq C\left\|\nabla_{h} b\right\|_{L^{2}}^{\frac{20 \alpha}{3(7 \alpha-15)}}\|\nabla b\|_{L^{2}}^{\frac{10 \alpha}{3(\alpha-15)}}\left\|u_{3}\right\|_{L^{\alpha}}^{\frac{10 \alpha}{4 \alpha-15}} .
$$

Integrating over interval $[\Gamma, \tau)$, it follows that

$$
\left\|b_{3}(\tau)\right\|_{L^{10}} \leq\left[\left\|b_{3}(\Gamma)\right\|_{L^{10}}^{\frac{10 \alpha}{4 \alpha-15}}+C \int_{\Gamma}^{\tau}\left\|\nabla_{h} b(\lambda)\right\|_{L^{2}}^{\frac{20 \alpha}{3(4 \alpha-15)}}\|\nabla b(\lambda)\|_{L^{2}}^{\frac{10 \alpha}{3(\alpha \alpha-15)}}\left\|u_{3}(\lambda)\right\|_{L^{\alpha}}^{\frac{10 \alpha}{4 \alpha-15}} d \lambda\right]^{\frac{4 \alpha-15}{10 \alpha}}
$$


for all $\tau \in[\Gamma, t)$. It follows from (3.7) and (3.5) that

$$
\begin{aligned}
& \mathcal{L}^{2}(t) \leq C+C \epsilon^{\frac{1}{4}} \mathcal{J}^{\frac{3}{2}}(t)\left(\int_{\Gamma}^{t}\left\|u_{3}(\tau)\right\|_{L^{\alpha}}^{\frac{8 \alpha}{3 \alpha-10}} d \tau\right)^{\frac{3 \alpha-10}{4(\alpha-2)}} \\
& +C \epsilon^{\frac{7}{8}} \mathcal{J}^{\frac{1}{4}}(t) \sup _{\tau \in[\Gamma, t]}\left[\left\|b_{3}(\Gamma)\right\|_{L^{10}}^{\frac{10 \alpha}{7 \alpha-15}}+C \int_{\Gamma}^{\tau}\left\|\nabla_{h} b(\lambda)\right\|_{L^{2}}^{\frac{20 \alpha}{3(4 \alpha-15)}}\|\nabla b(\lambda)\|_{L^{2}}^{\frac{10 \alpha}{3(\alpha-15)}}\left\|u_{3}(\lambda)\right\|_{L^{\alpha}}^{\frac{10 \alpha}{4 \alpha-15}} d \lambda\right]^{\frac{4 \alpha-15}{4 \alpha}} \\
& \leq C+C \epsilon^{\frac{1}{4}} \mathcal{J}^{\frac{3}{2}}(t)\left(\int_{\Gamma}^{t}\left\|u_{3}(\tau)\right\|_{L^{\alpha}}^{\frac{8 \alpha}{3 \alpha-10}} d \tau\right)^{\frac{3 \alpha-10}{4(\alpha-2)}} \\
& +C \epsilon^{\frac{7}{8}} \mathcal{J}^{\frac{1}{4}}(t)\left[\left\|b_{3}(\Gamma)\right\|_{L^{10}}^{\frac{10 \alpha}{4 \alpha-15}}+C \int_{\Gamma}^{t}\left\|\nabla_{h} b(\tau)\right\|_{L^{2}}^{\frac{20 \alpha}{3(4 \alpha-15)}}\|\nabla b(\tau)\|_{L^{2}}^{\frac{10 \alpha}{3(4 \alpha-15)}}\left\|u_{3}(\tau)\right\|_{L^{\alpha}}^{\frac{10 \alpha}{4 \alpha-15}} d \tau\right]^{\frac{4 \alpha-15}{4 \alpha}} \\
& \leq C+C \epsilon^{\frac{1}{4}} \mathcal{J}^{\frac{3}{2}}(t)\left(\int_{\Gamma}^{t}\left\|u_{3}(\tau)\right\|_{L^{\alpha}}^{\frac{8 \alpha}{3 \alpha-10}} d \tau\right)^{\frac{3 \alpha-10}{4(\alpha-2)}}+C\left\|b_{3}(\Gamma)\right\|_{L^{10}}^{\frac{5}{2}} \epsilon^{\frac{7}{8}} \mathcal{J}^{\frac{1}{4}}(t) \\
& +C \epsilon^{\frac{7}{8}} \mathcal{J}^{\frac{1}{4}}(t) \sup _{\tau \in[\Gamma, t]}\left\|\nabla_{h} b(\tau)\right\|_{L^{2}}^{\frac{5}{3}}\left[\int_{\Gamma}^{t}\|\nabla b(\tau)\|_{L^{2}}^{\frac{10 \alpha}{3(4 \alpha-15)}}\left\|u_{3}(\tau)\right\|_{L^{\alpha}}^{\frac{10 \alpha}{4 \alpha-15}} d \tau\right]^{\frac{4 \alpha-15}{4 \alpha}} \\
& \leq C+C \epsilon^{\frac{1}{4}} \mathcal{J}^{\frac{3}{2}}(t)\left(\int_{\Gamma}^{t} 1+\left\|u_{3}(\tau)\right\|_{L^{\alpha}}^{\frac{30 \alpha}{7 \alpha-45}} d \tau\right)^{\frac{3 \alpha-10}{4(\alpha-2)}}+C\left\|b_{3}(\Gamma)\right\|_{L^{10}}^{\frac{5}{2}} \epsilon^{\frac{7}{8}} \mathcal{J}^{\frac{1}{4}}(t) \\
& +C \epsilon^{\frac{7}{8}} \mathcal{J}^{\frac{1}{4}}(t) \mathcal{L}^{\frac{5}{3}}(t)\left[\left(\int_{\Gamma}^{t}\|\nabla b(\tau)\|_{L^{2}}^{2} d \tau\right)^{\frac{5 \alpha}{3(4 \alpha-15)}}\left(\int_{\Gamma}^{t}\left\|u_{3}(\tau)\right\|_{L^{\alpha}}^{\frac{3 \alpha \alpha}{7 \alpha-45}} d \tau\right)^{1-\frac{5 \alpha}{3(4 \alpha-15)}}\right]^{\frac{4 \alpha-15}{4 \alpha}} \\
& \leq C+C \epsilon^{\frac{1}{4}} \mathcal{J}^{\frac{3}{2}}(t)+C\left\|b_{3}(\Gamma)\right\|_{L^{10}}^{\frac{5}{2}} \epsilon^{\frac{7}{8}} \mathcal{J}^{\frac{1}{4}}(t)+C \epsilon^{\frac{7}{8}} \mathcal{J}^{\frac{1}{4}}(t) \mathcal{L}^{\frac{5}{3}}(t)\left[\epsilon+\int_{\Gamma}^{t}\left\|u_{3}(\tau)\right\|_{L^{\alpha}}^{\frac{30 \alpha}{7 \alpha-45}} d \tau\right]^{\frac{4 \alpha-15}{4 \alpha}} \\
& \leq C+C \epsilon^{\frac{1}{4}} \mathcal{J}^{\frac{3}{2}}(t)+C\left\|b_{3}(\Gamma)\right\|_{L^{10}}^{\frac{5}{2}} \epsilon^{\frac{7}{8}} \mathcal{J}^{\frac{1}{4}}(t)+C \epsilon^{\frac{7}{8}} \mathcal{J}^{\frac{1}{4}}(t) \mathcal{L}^{\frac{5}{3}}(t)\left[1+\int_{\Gamma}^{t}\left\|u_{3}(\tau)\right\|_{L^{\alpha}}^{\frac{30 \alpha}{7 \alpha-45}} d \tau\right]^{\frac{4 \alpha-15}{4 \alpha}} \\
& \leq C+C \epsilon^{\frac{1}{4}} \mathcal{J}^{\frac{3}{2}}(t)+C\left\|b_{3}(\Gamma)\right\|_{L^{10}}^{\frac{5}{2}} \epsilon^{\frac{7}{8}} \mathcal{J}^{\frac{1}{4}}(t)+C \epsilon^{\frac{21}{4}} \mathcal{J}^{\frac{3}{2}}(t)+\frac{5}{6} \mathcal{L}^{2}(t)
\end{aligned}
$$

which leads to

$$
\mathcal{L}^{2}(t) \leq C+C \epsilon^{\frac{1}{4}} \mathcal{J}^{\frac{3}{2}}(t)+C\left\|b_{3}(\Gamma)\right\|_{L^{10}}^{\frac{5}{2}} \epsilon^{\frac{7}{8}} \mathcal{J}^{\frac{1}{4}}(t)+C \epsilon^{\frac{21}{4}} \mathcal{J}^{\frac{3}{2}}(t) .
$$

Now, we will establish the bounds of $H^{1}$-norm of the velocity and magnetic field. In order to do it, we multiply the first and second equations of (1.1) by $-\Delta u$ and $-\Delta b$, respectively, and integrate them over $\mathbb{R}^{3}$ with respect to the spatial variable. Then, integration by parts gives the following identity:

$$
\begin{aligned}
& \frac{1}{2} \frac{d}{d t}\left(\|\nabla u\|_{L^{2}}^{2}+\|\nabla b\|_{L^{2}}^{2}\right)+\|\Delta u\|_{L^{2}}^{2}+\|\Delta b\|_{L^{2}}^{2} \\
= & -\sum_{i, j, k=1}^{3} \int_{\mathbb{R}^{3}} \partial_{k} u_{i} \partial_{i} u_{j} \partial_{k} u_{j} d x+\sum_{i, j, k=1}^{3} \int_{\mathbb{R}^{3}} \partial_{k} b_{i} \partial_{i} b_{j} \partial_{k} u_{j} d x-\sum_{i, j, k=1}^{3} \int_{\mathbb{R}^{3}} \partial_{k} u_{i} \partial_{i} b_{j} \partial_{k} b_{j} d x \\
& +\sum_{i, j, k=1}^{3} \int_{\mathbb{R}^{3}} \partial_{k} b_{i} \partial_{i} u_{j} \partial_{k} b_{j} d x
\end{aligned}
$$

Applying the divergence-free condition, $\nabla \cdot u=\nabla \cdot b=0$, by using the Hölder inequality, the 
interpolation inequality, and (3.1), it follows that

$$
\begin{aligned}
& \frac{1}{2} \frac{d}{d t}\left(\|\nabla u\|_{L^{2}}^{2}+\|\nabla b\|_{L^{2}}^{2}\right)+\|\Delta u\|_{L^{2}}^{2}+\|\Delta b\|_{L^{2}}^{2} \\
\leq & C \int_{\mathbb{R}^{3}}\left(\left|\nabla_{h} u\right|+\left|\nabla_{h} b\right|\right)\left(|\nabla u|^{2}+|\nabla b|^{2}\right) d x \\
\leq & C\left(\left\|\nabla_{h} u\right\|_{L^{2}}+\left\|\nabla_{h} b\right\|_{L^{2}}\right)\left(\|\nabla u\|_{L^{4}}^{2}+\|\nabla b\|_{L^{4}}^{2}\right) \\
\leq & C\left(\left\|\nabla_{h} u\right\|_{L^{2}}+\left\|\nabla_{h} b\right\|_{L^{2}}\right)\left(\|\nabla u\|_{L^{2}}^{\frac{1}{2}}\|\nabla u\|_{L^{6}}^{\frac{3}{2}}+\|\nabla b\|_{L^{2}}^{\frac{1}{2}}\|\nabla b\|_{L^{6}}^{\frac{3}{2}}\right) \\
\leq & C\left(\left\|\nabla_{h} u\right\|_{L^{2}}+\left\|\nabla_{h} b\right\|_{L^{2}}\right)\left(\|\nabla u\|_{L^{2}}^{\frac{1}{2}}\left\|\nabla_{h} \nabla u\right\|_{L^{2}}\|\Delta u\|_{L^{2}}^{\frac{1}{2}}+\|\nabla b\|_{L^{2}}^{\frac{1}{2}}\left\|\nabla_{h} \nabla b\right\|_{L^{2}}\|\Delta b\|_{L^{2}}^{\frac{1}{2}}\right) .
\end{aligned}
$$

Integrating this last inequality in time, we deduce that for all $\tau \in[\Gamma, t]$

$$
\begin{aligned}
\mathcal{J}^{2}(t) \leq & \|\nabla u(\Gamma)\|_{L^{2}}^{2}+\|\nabla b(\Gamma)\|_{L^{2}}^{2}+C \sup _{\tau \in[\Gamma, t]}\left(\left\|\nabla_{h} u(\tau)\right\|_{L^{2}}+\left\|\nabla_{h} b(\tau)\right\|_{L^{2}}\right) \\
& \times\left(\int_{\Gamma}^{t}\|\nabla u(\tau)\|_{L^{2}}^{2} d \tau\right)^{\frac{1}{4}}\left(\int_{\Gamma}^{t}\left\|\nabla \nabla_{h} u(\tau)\right\|_{L^{2}}^{2} d \tau\right)^{\frac{1}{2}}\left(\int_{\Gamma}^{t}\|\Delta u(\tau)\|_{L^{2}}^{2} d \tau\right)^{\frac{1}{4}} \\
& +C \sup _{\tau \in[\Gamma, t]}\left(\left\|\nabla_{h} u(\tau)\right\|_{L^{2}}+\left\|\nabla_{h} b(\tau)\right\|_{L^{2}}\right) \\
& \times\left(\int_{\Gamma}^{t}\|\nabla b(\tau)\|_{L^{2}}^{2} d \tau\right)^{\frac{1}{4}}\left(\int_{\Gamma}^{t}\left\|\nabla \nabla_{h} b(\tau)\right\|_{L^{2}}^{2} d \tau\right)^{\frac{1}{2}}\left(\int_{\Gamma}^{t}\|\Delta b(\tau)\|_{L^{2}}^{2} d \tau\right)^{\frac{1}{4}} \\
\leq & \|\nabla u(\Gamma)\|_{L^{2}}^{2}+\|\nabla b(\Gamma)\|_{L^{2}}^{2}+2 C \mathcal{L}(t) \epsilon^{\frac{1}{4}} \mathcal{L}(t) \mathcal{J}^{\frac{1}{2}}(t) \\
= & \|\nabla u(\Gamma)\|_{L^{2}}^{2}+\|\nabla b(\Gamma)\|_{L^{2}}^{2}+C \epsilon^{\frac{1}{4}} \mathcal{L}^{2}(t) \mathcal{J}^{\frac{1}{2}}(t) .
\end{aligned}
$$

Inserting (3.8) into (3.10) and taking $\epsilon$ small enough, then it is easy to see that for all $\Gamma \leq t<T^{*}$, there holds

$$
\begin{aligned}
\mathcal{J}^{2}(t) \leq & \|\nabla u(\Gamma)\|_{L^{2}}^{2}+\|\nabla b(\Gamma)\|_{L^{2}}^{2}+C \epsilon^{\frac{1}{4}} \mathcal{J}^{\frac{1}{2}}(t)+C \epsilon^{\frac{1}{2}} \mathcal{J}^{2}(t) \\
& +C\left\|b_{3}(\Gamma)\right\|_{L^{10}}^{\frac{5}{2}} \epsilon^{\frac{9}{8}} \mathcal{J}^{\frac{3}{4}}(t)+C \epsilon^{\frac{11}{2}} \mathcal{J}^{2}(t) \\
< & \infty
\end{aligned}
$$

which proves

$$
\sup _{\Gamma \leq t<T^{*}}\|\nabla u(t)\|_{L^{2}}^{2}+\|\nabla b(t)\|_{L^{2}}^{2}<\infty .
$$

This completes the proof of Theorem 1.1 .

\section{Aknoledgements}

This work was done while the second author was visiting the Catania University in Italy. He would like to thank the hospitality and support of the University, where this work was completed. This research is partially supported by P.R.I.N. 2019. The third author wish to thank the support of "RUDN University Program 5-100". 


\section{References}

[1] S. Benbernou, S. Gala and M. A. Ragusa, On the regularity criteria for the 3D magnetohydrodynamic equations via two components in terms of BMO space, Math. Methods Appl. Sci. 37 (2013), 2320-2325.

[2] J. Bergh and J. Löfström, Interpolation Spaces Springer, New York, 1976.

[3] X. Chen, S. Gala and Z. Guo, A new regularity criterion in terms of the direction of the velocity for the MHD equations, Acta Appl. Math. 113 (2011), 207-213.

[4] X. Chen, Z. Guo and M. Zhu, A new regularity criterion for the 3D MHD equations involving partial components, Acta Appl. Math. 134 (2014), 161-171.

[5] Q.L. Chen, C.X. Miao and Z.F. Zhang, On the regularity criterion of weak solution for the 3D viscous magnetohydrodynamics equations, Comm. Math. Phys. 284 (2008), 919-930.

[6] C. Cao and E.S. Titi, Regularity criteria for the three dimensional Navier-Stokes equations, Indiana Univ. Math. J. 57 (2008), 2643-2661.

[7] C. Cao and J. Wu, Two regularity criteria for the 3D MHD equations, J. Differential Equations 248 (2010), 2263-2274.

[8] B. Q. Dong, Y. Jia and W. Zhang, An improved regularity criterion of three-dimensional magnetohydrodynamic equations, Nonlinear Anal. RWA 13 (2012), 1159-1169.

[9] G. Duvaut and J. Lions, Inéquations en thermoélasticité et magnétohydrodynamique, Arch. Ration. Mech. Anal. 46 (1972), 241-279.

[10] S. Gala, Extension criterion on regularity for weak solutions to the 3D MHD equations, Math. Methods Appl. Sci. 33 (2010) 1496-1503.

[11] S. Gala and M.A. Ragusa, A new regularity criterion for the 3D incompressible MHD equations via partial derivatives, J. Math. Anal. Appl. 481, Issue 2 (2020), 123497.

[12] S. Gala and M.A. Ragusa, On the regularity criterion of weak solutions for the 3D MHD equations, Z. Angew. Math. Phys. 68 (2017), 140.

[13] S. Gala and M.A. Ragusa, A logarithmic regularity criterion for the two-dimensional MHD equations, J. Math. Anal. Appl. 444, Issue 2 (2016), 1752-1758.

[14] C. He and Z. Xin, On the regularity of weak solutions to the magnetohydrodynamic equations, J. Differential Equations, 213 (2005), 235-254.

[15] C. He and Y. Wang, Remark on the regularity for weak solutions to the magnetohydrodynamic equations, Math. Methods Appl. Sci. 31 (2008), 1667-1684.

[16] E. Ji and J. Lee, Some regularity criteria for the 3D incompressible magnetohydrodynamics, J. Math. Anal. Appl. 369 (2010), 317-322. 
[17] X. Jia and Y. Zhou, Regularity criteria for the 3D MHD equations involving partial components, Nonlinear Anal. RWA 13 (2012), 410-418.

[18] X. Jia and Y. Zhou, Regularity criteria for the 3D MHD equations via partial derivatives, Kinet. Relat. Models 5 (2012), 505-516.

[19] X. Jia and Y. Zhou, Regularity criteria for the 3D MHD equations via partial derivatives, II. Kinet. Relat. Models 7 (2014), 291-304.

[20] X. Jia and Y. Zhou, Ladyzhenskaya-Prodi-Serrin type regularity criteria for the 3D incompressible MHD equations in terms of 33 mixture matrices, Nonlinearity 28 (2015), 3289-3307.

[21] X. Jia and Y. Zhou, On regularity criteria for the 3D incompressible MHD equations involving one velocity component, J. Math. Fluid Mech.18 (2016), 187-206.

[22] G.G. Lorentz, Some new functional spaces, Annals of Mathematics 51 (1950), 37-55.

[23] L. Ni, Z. Guo and Y. Zhou, Some new regularity criteria for the 3D MHD equations, J. Math. Anal. Appl. 396 (2012), 108-118.

[24] R. O’Neil, Convolution operators and $L^{p, q}$ spaces, Duke Math. J. 30 (1963), 129-142.

[25] M. Sermange and R. Temam, Some mathematical questions related to the MHD equations, Comm. Pure Appl. Math. 36 (1983), 635-664.

[26] H. Triebel, Theory of Function Spaces. Basel: Birkhäuser, 1983.

[27] J. Wu, Viscous and inviscid magnetohydrodynamics equations, J. Anal. Math. 73 (1997), 251-265.

[28] J. Wu, Bounds and new approaches for the 3D MHD equations, J. Nonlinear Sci. 12 (2002), 395-413.

[29] J. Wu, Regularity results for weak solutions of the 3D MHD equations, Discrete Contin. Dyn. Syst. 10 (2004), 543-556.

[30] Y. Zhou, Remarks on regularities for the 3D MHD equations. Discrete Contin. Dyn. Syst. 12 (2005), 881-886.

[31] K. Yamazaki, Regularity criteria of MHD system involving one velocity and one current density component, J. Math. Fluid Mech. 16 (2014), 551-570.

[32] K. Yamazaki, Regularity criteria of the three-dimensional MHD system involving one velocity and one vorticity component, Nonlinear Anal. 135 (2016), 78-83.

[33] K. Yamazaki, Remarks on the regularity criteria of the three-dimensional magnetohydrodynamics system in terms of two velocity field components. J. Math. Phys. 55 (2014), 031505, 16 pp. 
[34] Z. Zhang, Regularity criteria for the 3D MHD equations involving one current density and the gradient of one velocity component, Nonlinear Anal. 115 (2015), 41-49.

[35] Z. Zhang, Remarks on the global regularity criteria for the 3D MHD equations via two components, Z. Angew. Math. Phys., 66 (2015), 977-987.

[36] Z. Zhang, Re ned regularity criteria for the MHD system involving only two components of the solution, Appl. Anal. 96 (2017), 2130-2139.

[37] Y. Zhou and S. Gala, Regularity criteria for the solutions to the 3D MHD equations in the multiplier space, Z. Angew. Math. Phys. 61 (2010), 193-199.

[38] Y. Zhou and S. Gala, A new regularity criterion for weak solutions to the viscous MHD equations in terms of the vorticity field, Nonlinear Anal. 72 (2010), 3643-3648. 